\title{
Wrist-gathered acceleration data and their correlation with physical activity in the elderly
}

\author{
Amy Papadopoulos ${ }^{1,}$, Nicolas Vivaldi ${ }^{1,2}$, Cindy Crump ${ }^{1,3}$, Christine Tsien Silvers ${ }^{1,4}$ \\ ${ }^{1}$ AFrame Digital, Inc., Vienna, VA, USA \\ ${ }^{2}$ Department of Biomedical Engineering, George Washington University, Washington, DC, USA \\ ${ }^{3}$ The Center for Study of Chronic Illness and Disability, George Mason University, Fairfax, VA, USA \\ ${ }^{4}$ Children's Hospital Informatics Program, Children's Hospital, Boston, MA, USA
}

\section{Email address:}

amylingley2@aol.com (A. Papadopoulos),nvivaldi@gwmail.gwu.edu (N. Vivaldi), ccrump@aframedigital.com (C. Crump), tsien@alum.mit.edu (C. T. Silvers)

\section{To cite this article:}

Amy Papadopoulos, Nicolas Vivaldi, Cindy Crump, Christine Tsien Silvers. Wrist-Gathered Acceleration Data and their Correlation with Physical Activity in the Elderly. International Journal of Biomedical Science and Engineering. Vol. 2, No. 5, 2014, pp. 38-44.

doi: $10.11648 /$ j.ijbse.20140205.11

\begin{abstract}
Estimating physical activity in the elderly from wrist-gathered acceleration data was studied. Thirty individuals (65+ years) were video-recorded while wearing a wrist device and going about their normal activities within their regular living environment for four hours each. Acceleration data were summarized into an activity value [via the "differential signal magnitude" (DSM) method] and compared to metabolic equivalent of task (MET) values determined by video analysis for each time period ("epoch"). Different sampling rates and epoch sizes were evaluated. Sampling at $4 \mathrm{~Hz}$ and using 60 -second epochs provided the best results, with a moderate Pearson's correlation coefficient of 0.58 between DSM activity values and MET values. The area under the receiver operating characteristic curve (AUC) for classifying each minute of data as active (MET $>=2.0$ ) versus moderately active (MET > 1.2 and $<2.0$ ) was 0.87 (sensitivity $80 \%$, specificity $79 \%$ ). DSM activity values (sampling at 4 $\mathrm{Hz}$ ) were compared to the widely known signal magnitude area (SMA) values (requiring low-pass filtering and sampling at 40 $\mathrm{Hz}$ ), with an excellent correlation of 0.994 .
\end{abstract}

Keywords: Accelerometer, Activity Level, Differential Signal Magnitude, MET Values, Metabolic Equivalent of Task, Physical Activity Level, Tri-Axial Accelerometer, Wearable Sensors

\section{Introduction}

A substantial amount of research on human activity monitoring has been undertaken. Various systems have been tested-ranging from accelerometer and gyroscope sensors to video camera - to detect dangerous situations (e.g., falls) and signal help if necessary. Physical activity monitoring can provide a great deal of health-related information about an individual; in fact, Healthy People 2020, an organization under the U.S. Department of Health and Human Services, identified physical activity as a leading health indicator [1]. The health of a person can be evaluated on the basis of his/her activity levels in comparison to baseline values or on his/her ability to perform basic actions. To be practical for elderly patients living independently, such systems cannot be cumbersome or expensive, and should require little to no self-maintenance.

Surveys of activity are perhaps the simplest health and activity evaluation method, but they are limited in scope and are prone to biased reporting, which can lead to inaccurate results. The National Health and Nutritional Examination Survey from 2003-2004 noted as much when they paired survey data with week-long accelerometer trials for over 7000 participants aged 6-60+ in order to determine adherence to recommended activity levels. Although the study did not focus on monitoring methods, data collected did show the mentioned weaknesses of stand-alone surveys [2].

The earliest use of an accelerometer for measurement of human energy expenditure was by Wong et al in 1981 [3]. They conducted a study to determine whether or not acceleration data from a uni-axial accelerometer integrated over 24 hours would properly correlate to oxygen consumption, from which energy expenditure can be calculated. When compared to other available technologies, the accelerometer showed the greatest efficacy in correlating with measured oxygen consumption, leading the researchers 
to conclude that accelerometer-based devices are prime candidates for measurement of physical activity and energy expenditure.

More recently, researchers have looked at using multiple sensors and/or sensors at multiple locations on the body when analyzing activity. For example, Gjoreski et al [4] attempted to use different combinations of four tri-axial accelerometers together with a location sensor system to determine posture as a way of detecting increased probability of falls. Nine combinations of accelerometer placements (on the chest, waist, right ankle, and thigh) in addition to four location tags were tested for their ability to correctly determine posture. Likewise Yeoh et al [5] used three bi-axial accelerometers, one on the waist and one on each thigh, to determine posture and walking speed as a means of identifying activity. When determining posture, an Extended Kalman filtering algorithm was used in a heuristic classification system. With five test subjects, the system correctly identified walking, sitting, standing, and lying $100 \%$ of the time in 40 tasks ordered randomly

Even though many studies focus on multiple sensor systems, these arrangements are not practical. Systems with multiple sensors across the body are more complicated than single-sensor systems, and are more likely to deter patients from using them. Based on the need for convenience, Bieber $e t$ al [6] conducted a study using the accelerometers embedded within the Sony Ericsson w715 mobile phones; they were able to successfully detect stand-up and sit-down transitions. Similarly, Kang et al [7] conducted a study that used a single tri-axial accelerometer mounted on a belt around the waist. Ten subjects performed a range of activities (falls, walking/running, and posture transitions) while wearing the device; an algorithm was then used to differentiate amongst the different activities. The system had an overall recognition rate of $96.1 \%$. While these studies produced good results, Bassett et al [8] stated that energy expenditure (in activities other than walking) is typically under-predicted by motion sensors due to their inability to measure arm movement. A sensor placed at the wrist, however, would not have this same limitation. In addition, a wrist device would be socially acceptable due to its resemblance to watches, wristbands, etc., unlike the devices suggested for placement on the waist, legs, or trunk.

Stiles et al [9] studied the use of accelerometers worn at either the hip or wrist and found either location can be used to classify activity that is beneficial to bone in premenopausal women. Zhang et al [10] experimented with various sampling frequencies and number of axes when using accelerometer data from the wrist to classify activity level in adults (average age 49.4). Ekblom et al [11] reported on promising results for using wrist accelerometry to estimate energy expenditure and physical activity in children aged 8 to 10 years.

The method used for analyzing the data is just as important as the device itself, and likewise there are many techniques for performing such analysis. Figo et al [12] recently sought to evaluate competing methods both qualitatively and quantitatively on the basis of algorithmic complexity, memory requirements, and accuracy of identifying activity in an experimental setting. The methods were grouped into three categories: time domain, frequency domain, and discrete domain. It was found that frequency-domain techniques were more suited for identifying walking, running, and jumping when the three activities were performed consecutively, but that time-domain techniques were simpler and yielded higher accuracy when only discriminating between two such events.

Bouten et al [13] was the first to introduce a technique known as the signal magnitude area (SMA) method, which uses the area under the acceleration curve as a measure of activity. SMA has become widely used as a processing technique for accelerometer data. The values it generates can be used to distinguish different levels of activity, as well as relate to energy expenditure $[14,15,16]$. Khan et al [17] showed that by using SMA to augment the Autoregressive model (AR), the accuracy of characterizing both posture and physical activity dramatically increases. Furthermore, Bassett et al [8] studied the validity of using SMA in real-time activity monitoring by applying SMA to data collected from a waist-mounted accelerometer and comparing the results to metabolic equivalent (MET) values determined through use of a device that measured oxygen consumption.

During this study, as a means of validating classification and characterization of an older adult's activity in real time using a nonintrusive wearable wrist sensor, data analysis was performed on activity and video data collected during an IRB-approved protocol. The study goal was to create an automated algorithmic classification of activities performed by the elderly in routine daily life (running, sitting, sleeping, reading, eating, etc.) into one of three activity level groupings (inactive, moderately active, active). The approach uses an algorithm similar to-but more efficient than-SMA, requiring fewer samples and not requiring use of a low-pass filter. The activity values determined using this algorithm were compared to MET values assigned by manual video analysis as well as to the values determined using the well-established SMA method.

\section{Methods}

\subsection{Data Collection}

Thirty elderly (aged 65 and over), ambulatory volunteers residing in an independent living facility were recruited and gave their consent to an IRB-approved protocol. The volunteers were asked to engage in normal activities of daily living (ADLs) while each being monitored over a four-hour time period (from 11 a.m. to 3 p.m.). The volunteers were video-recorded while data from a noninvasive, wireless wrist device containing a tri-axial accelerometer were recorded (see Fig. 1). There were no limitations on the activities in which the participants were allowed to engage other than that they were asked not to ride in a bus, car, or other vehicle, and not to engage in water-submersion activities (e.g., swimming).

The wrist accelerometer was sampled at 125 samples/second. Each sample consisted of three bytes of data. Each byte represented data from one of the three 
accelerometer axes. The range of the samples was $+/$ - eight times the acceleration due to gravity $(\mathrm{g})$. The accelerometer functionality was such that when no movement was present, the normalized magnitude would be $1 \mathrm{~g}$. A timestamp, which was to the nearest second, was available for the beginning and ending of data collection.

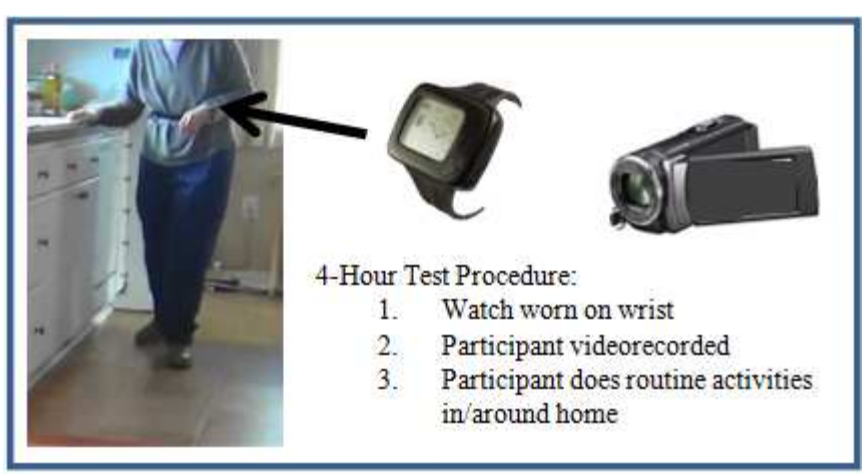

Figure 1. Data collected for each study participant consisted of accelerometry data from a watch device worn on the wrist and videorecording data from a research assistant-held videocamera over a 4-hour period as the participant went about his/her normal activities of daily living.

\subsection{Video Summaries}

De-identified video summaries that could be read into MATLAB for analysis were manually created for each video, with times in the summary given relative to the beginning of each video and provided to the nearest second. The summaries included a number of items that were used to help synchronize the video with the wrist data, such as the second when the data capture was started and stopped. In addition, each summary had rows for standing and sitting that represented the second the person reached either a standing or sitting position. Subcategories under standing and sitting were also included and listed when specific activities started and stopped. Some activities - which were of short duration, hard to define, or very infrequent - were marked as "standing other" or "sitting other." If the person was not doing something that fit into one of the subcategories, that second of data was not specified separately. Periods of sitting or standing that were not marked as any particular type of activity were spent in conversation or indicated sitting or standing still.

Activities that were included as subcategories of standing were: walking, going up stairs, going down stairs, working in the kitchen, doing carpentry, ironing, dressing, doing the laundry, washing, talking on the phone, bending down, watering plants, and making the bed. Activities that were included as subcategories of sitting were: doing paperwork, doing a puzzle, reading, writing, using a computer, eating or drinking, talking on the phone, playing the piano, knitting, sleeping, shining shoes, wrapping gifts, doing electrical repairs, sewing, and getting a massage.

A list of MET values published by Ainsworth et al [18] was used to determine the MET value associated with each activity listed as a subcategory, as well as with the general categories of sitting and standing. A MET is a standard physiological measure describing the amount of energy required by specific activities. One MET is defined as the amount of energy required to sit still (i.e., the resting metabolic rate obtained during quiet sitting). The MET values associated with each activity were used to assign a MET level to each time period ('epoch') of activity. When multiple activities were present in an epoch, the single activity type present for the greatest number of seconds was used as the activity type for that epoch.

\subsection{Data Analysis}

The activity data gathered and stored by the wrist device were manipulated using MATLAB to create a single measurement of activity for each epoch. During the analysis, the optimal epoch length was also evaluated. The lengths considered were $30,60,90$, and 120 seconds. The lengths under consideration were selected to be the same as the periods already available on the wrist device for communication with the database server. In order to preserve network bandwidth, the goal was to develop an activity measurement that could be inserted into an already existing message being sent between the measurement device and the server.

In addition, the optimal number of samples per second used to determine the activity measurement was evaluated. While the data were sampled at 125 samples per second during data collection, this sampling rate would not be reasonable for constant use in a real-time, data-streaming application without creating performance issues. Higher sampling rates would be detrimental to the battery life of the wearable device, which was designed to be worn for many days between charges in order to be practical, socially acceptable, and less burdensome for the wearer. Lower sampling rates, on the other hand, might not provide enough information. The sampling rates considered during the project were one, two, four, and eight samples per second, and one sample per every four and eight seconds.

The only pre-processing step used was a median filter of length five samples. The method used to aggregate the data combines the ideas behind SMA and the differential signal vector magnitude (DSVM) method [9]. The equation, which can be referred to as the differential signal magnitude (DSM) method, is shown in (1).

The DSM method was developed so that a low-pass filter, as necessary when using SMA, would not be required. Additionally, most implementations of the SMA method use a higher sampling rate (of about $40 \mathrm{~Hz}$ ) than the sampling rate desired for this application.

$$
D S M=\frac{1}{n} \sum_{1}^{n}\left(\left|x_{n}-x_{n-1}\right|+\left|y_{n}-y_{n-1}\right|+\left|z_{n}-z_{n-1}\right|\right)
$$

The originally collected data streams were sampled using the various sampling rates under consideration as indicated and then combined using the DSM method over the various epochs under consideration to provide arrays representing activity level over time (see Fig. 2). The MET values mapped to each epoch were then compared to the calculated DSM 
activity levels, and Pearson correlation coefficients were derived for each of the sampling rate/epoch combinations. The Pearson correlation coefficient is a measure of the linear dependence between two variables, or in simpler terms, the degree to which the two variables form a line in two-dimensional space. Values close to +1 indicate a direct relationship between the two variables, values close to -1 indicate an indirect relationship between the two variables, and values close to 0 indicate a weak relationship between the two variables. As the absolute value of the correlation coefficient moves from 0 to 1 , the strength of the linear relationship between the two variables under consideration increases.

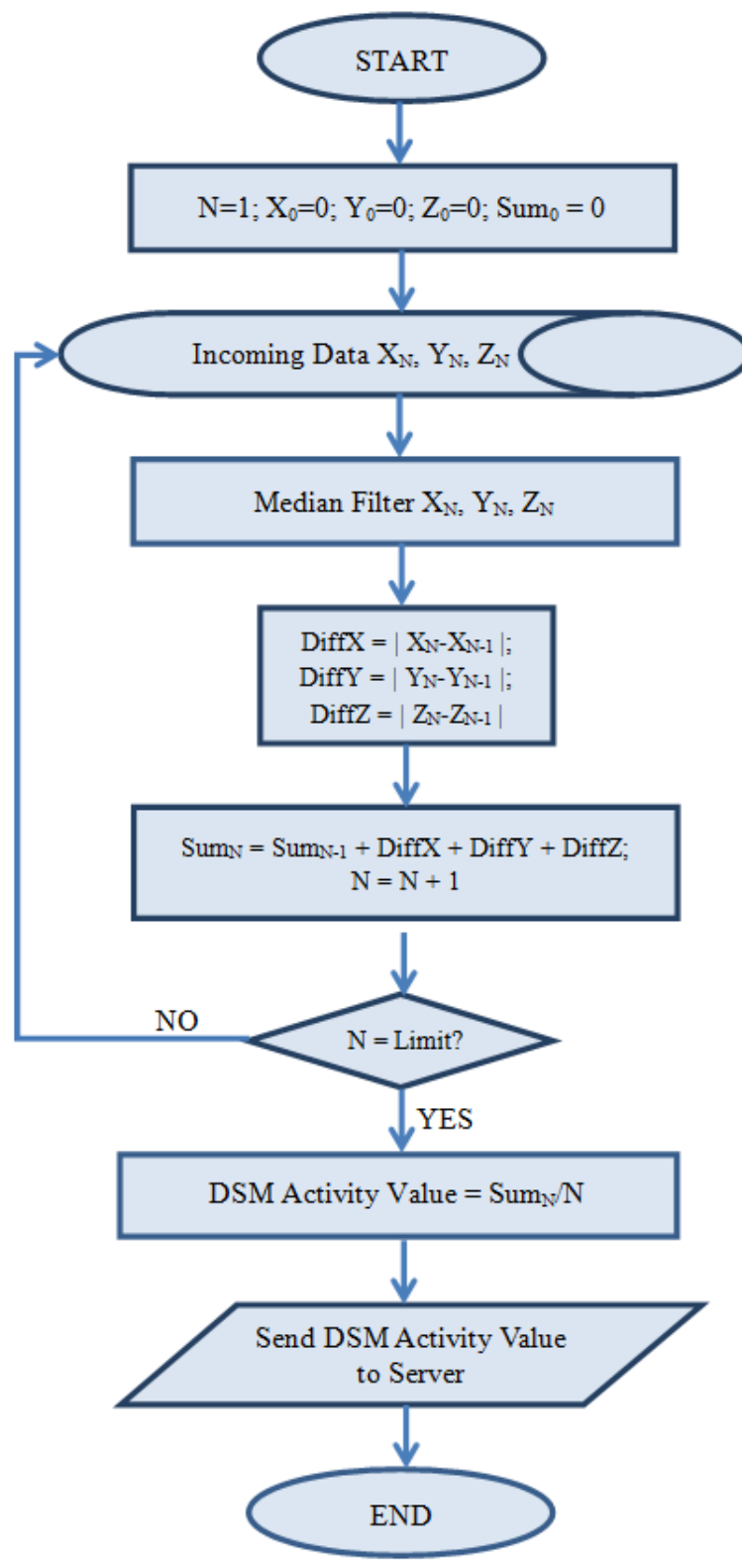

Figure 2. This flowchart outlines the data processing involved, from accelerometer measurements $(X, Y, Z)$ to the differential signal magnitude (DSM) activity sum value $[N=$ Count, Limit $=$ Number of Samples Per Second $X$ Number of Seconds Per Activity Value, DSM = Differential Signal Magnitude].
The sampling rate and epoch with the highest correlation coefficient were selected for further analysis. The ability of the DSM method to differentiate between different levels of activity was tested by creating a receiver operating characteristic (ROC) curve, from which to then calculate the area under that curve (AUC).

In order to determine the best thresholds for distinguishing between inactivity, moderate activity, and activity, a linear regression line representing the relationship between the DSM values and the MET values was created. The activity value corresponding to 2 METs on the regression line was used as a threshold between active and moderately active and the value corresponding to 1.2 METs on the regression line was used as a threshold between moderately active and inactive. The sensitivity and specificity values achieved when these selected activity values were used as thresholds were calculated.

As a means of validating the DSM method, which the authors have recently noted was also developed and tested completely independently by Carus et al [19], the data were processed using the SMA method, both with and without use of a low-pass filter, and with sampling at both 4 and $40 \mathrm{~Hz}$. These results were then compared to the DSM results. As a final step, the correlation coefficient between the values resulting from the DSM method with sampling at $4 \mathrm{~Hz}$ and the values resulting from the SMA method using a low-pass filter and sampling at $40 \mathrm{~Hz}$ was calculated.

\section{Results}

The correlation coefficients between the DSM values and the MET values were calculated for each sampling rate/epoch combination (Fig. 3). The correlation coefficients varied between 0.38 and 0.60 with a very high level of significance (a $p$-value of $<0.0005)$. The highest correlation coefficients, in the range of 0.54 to 0.60 for all epochs tested, occurred when sampling at four samples per second. When considering the ideal epoch length, there was very little difference between epoch lengths of 60,90 , and 120 seconds. The results for the 30 -second epoch length were not quite as good as those for the longer epochs. All of the correlation coefficients when sampling at four samples per second for epochs of 60,90 , and 120 seconds were in the range of 0.58 to 0.60 . These results indicate that while using a sampling rate of four samples per second and an epoch of 60,90 , or 120 seconds, activity values derived by the DSM method indeed correlate with MET values. Since there was very little difference between the results for epochs of 60, 90, and 120 seconds, an epoch length of 60 seconds was used for the remaining calculations.

After the sampling rate and time period were selected, the ability of the DSM method to differentiate between different levels of activity was tested by calculating the AUC. The AUC when differentiating active (MET $>=2$ ) from moderately active $(1.2<$ MET $<2.0)$ ADLs was 0.86 . There were not enough data from the study participants to create an ROC curve for differentiating moderately active from inactive (MET <=1.2) ADLs. 


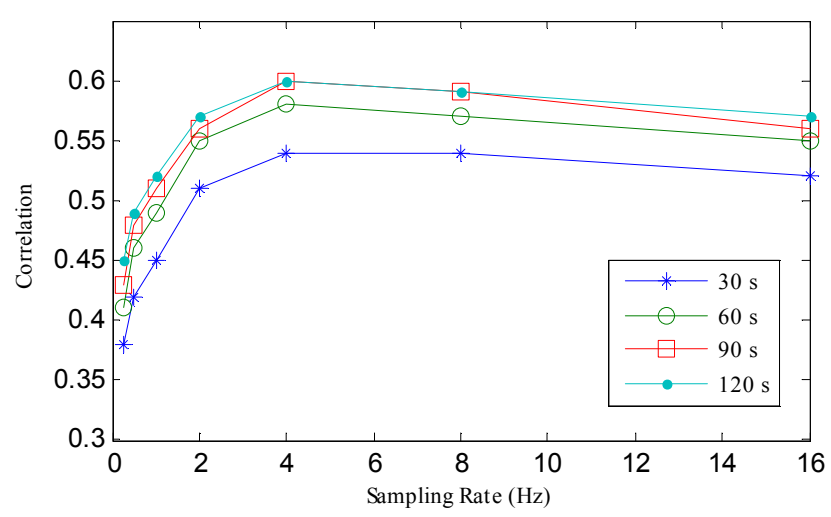

Figure 3. Effect of sampling rate and epoch size on correlation between DSM and MET values [DSM = differential signal magnitude, $M E T=$ metabolic equivalent, $\mathrm{Hz}=$ Hertz, $\mathrm{s}=$ second].

The linear regression line representing the relationship between DSM and MET values is shown in Fig. 4. The equation of the line is $y=22.6 x-13.6$. Based on this equation, the DSM threshold between moderately active and inactive would fall at about 14 and the threshold between active and moderately active would fall at about 32 . Using the value of 32 to differentiate between active and moderately active values using the DSM method, the sensitivity was $71 \%$ and the specificity was $84 \%$. These numbers improved slightly as the threshold was lowered to 28 , at which point the sensitivity was $80 \%$ and the specificity $79 \%$. There were not enough data in the inactive category to determine the sensitivity and specificity of differentiating between moderately active and inactive ADLs.

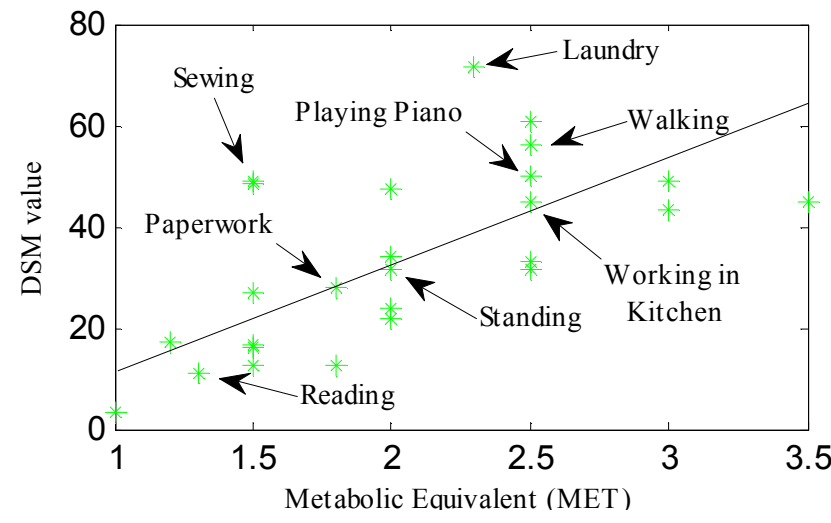

Figure 4. Relationship between average DSM activity values for specific activity types and MET values. Each data point represents a specific activity. The line going through the graph is the linear regression line based on all data points and their known MET values [DSM = differential signal magnitude, $M E T=$ metabolic equivalent $]$.

Table 1 shows the results when comparing the DSM method to the SMA method. As mentioned, the SMA method is usually used after pre-processing data, sampled at 40 or more samples per second, with a low-pass filter. When using the SMA method with a filter and the suggested sampling rate, the correlation with METs is the same as when using the DSM method. However, if using the SMA method in the same manner as the DSM method, that is, with a lower sampling rate and without a low-pass filter, the SMA results are significantly worse. Finally, the correlation between the DSM method using four samples per second and no low-pass filter and the SMA method using 40 samples per second and a low-pass filter is 0.994 .

Table 1. Comparison of the DSM and SMA methods with and without use of a low-pass filter and using both four and 40 samples per second [DSM = differential signal magnitude, SMA = signal magnitude area, MET = metabolic equivalent, $A U C=$ area under the receiver operating characteristic curve, $\mathrm{Hz}=$ Hertz].

\begin{tabular}{lll}
\hline Calculation Method & $\begin{array}{l}\text { Correlation } \\
\text { with MET }\end{array}$ & $\begin{array}{l}\text { AUC } \\
\text { Moderately Active } \\
\text { Morsus }\end{array}$ \\
\hline DSM 4 Hz, no filter & 0.580 & 0.858 \\
SMA 4 Hz, no filter & 0.053 & 0.560 \\
SMA 4 Hz, low-pass filter & 0.303 & 0.684 \\
SMA 40 Hz, no filter & 0.055 & 0.570 \\
SMA 40 Hz, low-pass filter & 0.580 & 0.859 \\
\hline
\end{tabular}

\section{Discussion}

During this study, data analysis was performed on tri-axial accelerometer data gathered from 30 individuals aged 65 and over while they were being simultaneously video-recorded in their usual living environment in order to determine whether data gathered from the socially-acceptable wrist location could be used to provide an activity value that correlates with physical activity values. The study considered the optimal sampling rate and epoch length, as well as made use of an algorithm that sums the differences between successive samples, referred to as the DSM method, in order to derive an activity measure. The results of the study indicate that the DSM method offers a means of providing a value for physical activity that is moderately correlated with video analysis-assigned MET value estimates, and highly correlated with the well-established SMA method for estimating physical activity and energy expenditure in older adults going about their regular ADLs. Different sampling rates were tested, with a sampling rate of four samples per second providing the best results. Likewise, different epochs were tested, with the conclusion that epochs of 60, 90, and 120 seconds all produced very similar results. When using four samples per second and an epoch of 60 seconds, the correlation between the output of the DSM method and accepted MET values was 0.58. Furthermore, the AUC resulting from differentiating between active (MET $>=2.0)$ and moderately active $(1.2<$ MET < 2.0) ADLs was 0.86.

There are two limitations of this study. The first is that the comparison MET values were based on estimates by published activity categories [18] assigned to each second of the data by analysis of corresponding video recordings. While the analysis was carefully and manually performed on a second-to-second basis, the comparison values still represent estimates in themselves. This method of determining MET values used in the present study was chosen because the video summaries and matching acceleration data had already been collected for a previous research study. A desirable next step would be to confirm the correlation between the calculated DSM activity values and MET values in a follow-on study that 
measures oxygen consumption with a portable measuring device. It is worth noting, however, that such an equipment setup would likely be considered somewhat intrusive, cumbersome, and uncomfortable, making it more difficult to recruit the necessary subjects, especially amongst older adults.

The other limitation of this study is that there were not enough data to create an ROC curve for differentiating between moderately active and inactive ADLs. A follow-on study should be designed to concentrate on activities at the lower end of the activity scale, possibly including nap times or sleep periods. Future work should also include analysis of correlation between DSM values and MET values for activities in those younger than age 65 .

Despite these limitations, the results independently confirm the findings of Carus et al [19] that the DSM method provides values that are highly correlated with the well-established SMA values, without the costs associated with the use of a higher sampling rate and a low-pass filter. The correlation measured between the two methods on the provided dataset was 0.994, indicating near-perfect correlation. As Carus et al mentioned, a number of studies have shown that SMA values are correlated with METs (as determined using gold-standard techniques for measuring MET values); therefore, the high correlation between DSM values and SMA values here strongly supports that DSM activity values are likewise correlated with MET values.

\section{Conclusion}

There are two aspects of this research that are key to the success of systems designed to measure activity in real time as individuals go about their normal lives. The first is that the data are gathered at the wrist location. A sensor at the wrist location, especially one in watch form factor, is much more socially acceptable than a sensor placed at the waist, hip, and/or trunk. In addition, a wrist sensor in watch form factor is likely more comfortable than sensors placed in other body locations, allowing a wrist sensor to be worn 24/7, including during periods of sleep. The second aspect is that the DSM method requires less computation and fewer samples than the well-established SMA method, while providing nearly the same results. Less computation and fewer samples will result in improved battery life, a trait that is necessary for any system that hopes to be successful for mobile monitoring of physical activity.

\section{Acknowledgments}

The authors would like to thank the staff at Vinson Hall Retirement Community for their assistance with participant recruitment and data collection, and the residents at Vinson Hall Retirement Community for their participation.

The data used for this analysis were collected during a study supported by Grant Number R43AG039176 from the National Institute on Aging. The content is solely the responsibility of the authors and does not necessarily represent the official views of the National Institute on Aging or the National
Institutes of Health.

The data analysis was supported by CRCF Award SBIR-012 from the Commonwealth of Virginia Small Business Innovation Research grant "Commonwealth Research and Commercialization Fund" Matching Award from the Commonwealth of Virginia.

All authors at some point were employed by and/or held stock options in AFrame Digital, Inc., the company that developed the wrist device used to gather the acceleration data.

\section{References}

[1] Healthy People 2020, HealthyPeople.gov, managed by the U.S. Department of Health and Human Services, Washington, DC, last updated February 6, 2013.

[2] R. Troiano, D. Berrigan, K. Dodd, L. Masse, T. Tilert, and M. McDowell, "Physical activity in the United States measured by accelerometer," Medicine \& Science in Sports \& Exercise, pp. 181-188, 2007.

[3] T. Wong, J. Webster, H. Montoye, and R. Washburn, "Portable accelerometer device for measuring human energy expenditure,” IEEE Trans. Biomed. Eng., vol. 28, pp. 467-471, 1981.

[4] H. Gjoreski, M. Lustrek, and M. Gams, "Accelerometer placement for posture recognition and fall detection," 2011 Seventh International Conference on Intelligent Environments, pp. 47-54, 2011.

[5] W. Yeoh, I. Pek, Y. Yong, X. Chen, and A. Waluyo, "Ambulatory monitoring of human posture and walking speed using wearable accelerometer sensors," $30^{\text {th }}$ Annual International IEEE EMBS Conference, Vancouver, British Columbia, Canada, pp. 5184-5187, August, 2008.

[6] G. Bieber, P. Koldrack, C. Sablowski, C. Peter, and B. Urban, "Mobile physical activity recognition of stand-up and sit-down transitions for user behavior analysis," PETRA 2010, Samos, Greece, June 2010.

[7] D. Kang, J. Choi, G. Tack, B. Lee, J. Lee, S. Chung, and S. Park, "Real-time elderly activity monitoring system based on a tri-axial accelerometer," Proceedings of the $2^{\text {nd }}$ International Convention on Rehabilitation Engineering \& Assistive Technology, pp. 262-265, Singapore, 2008.

[8] D. Bassett, B. Ainsworth, A. Swartz, S. Strath, W. O'Brien, and G. King, "Validity of four motion sensors in measuring moderate intensity physical activity," Med Sci Sports Exerc., pp. S471-S480, 2000.

[9] V.H. Stiles, P.J. Griew, A.V. Rowlands, "Use of accelerometry to classify activity beneficial to bone in premenopausal women," Med Sci Sports Exerc., vol. 45(12), pp. 2353-2361, 2013.

[10] S. Zhang, P. Murray, R. Zillmer, R.G. Eston, M. Catt, A.V. Rowlands, "Activity classification using the GENEA: optimum sampling frequency and number of axes," Med Sci Sports Exerc., vol. 44(11), pp. 2228-2234, 2012.

[11] O. Ekblom, G. Nyberg, E.E. Bak, U. Ekelund, C. Marcus, "Validity and comparability of a wrist-worn accelerometer in children," J Phys Act Health, vol. 9(3), pp. 389-393, 2012. 
[12] D. Figo, P. Diniz, D. Ferreir, J. Cardoso, "Preprocessing techniques for context recognition from accelerometer data," Personal and Ubiquitous Computing, vol. 14(7), pp. 645-662, 2010.

[13] C. Bouten, K. Westerterp, M. Verduin, and J. Janssen, "Assessment of energy expenditure for physical activity using a triaxial accelerometer," Med. Sci. Sports Exerc., vol. 26, pp. 1516-1523, 1994

[14] D. Karantonis, M. Narayanan, M. Mathie, N. Lovell, and B. Cellar, "Implementation of a real-time human movement classifier using a triaxial accelerometer for ambulatory monitoring," IEEE Transactions on Information Technology in Biomedicine, vol. 10(1), pp. 156-167, 2006.

[15] A. Jeon et al., "Implementation of the personal emergency response system," International Journal of Biological and Medical Sciences, vol. 1(1), pp. 61-65, 2008.
[16] C. Bouten, W. Verboeket-Van De Venne, K. Westerterp, M. Verduin, and J. Janssen, "Daily physical activity assessment: comparison between movement registration and doubly labeled water," The American Physiological Society, pp. 1019-1026, 1996.

[17] A. Khan, Y. Lee, and T. Kim, "Accelerometer signal-based human activity recognition using augmented autoregressive model coefficients and artificial neural nets," $30^{\text {th }}$ Annual International IEEE EMBS Conference, Vancouver, British Columbia, Canada, pp. 5172-5175, August, 2008.

[18] B. Ainsworth et al., "Compendium of physical activities: an update of activity codes and MET intensities," Med Sci Sports Exerc., pp. S498-S516, 2000.

[19] J.L. Carus, V. Pelaez, G. Lopez, and V. Lobato, “JIM: a novel and efficient accelerometric magnitude to measure physical activity," pHealth, pp. 283-288, 2012. 